\title{
QD Comparação de métodos de estimativa da evapotranspiração de referência no período chuvoso e seco em Piripiri (PI)
}

Aureliano Albuquerque Ribeiro ${ }^{1}$

Marcelo Simeão ${ }^{2}$

Ananda Rosa Beserra Santos 3

\section{Resumo}

Diversos métodos empíricos vêm sendo utilizados para a quantificação da evapotranspiração de referência em virtude das dificuldades de acesso dos produtores às variáveis empregadas pelo modelo-padrão de Penman-Monteith-FAO. Assim, objetivou-se com a realização deste trabalho avaliar, para as condições climáticas de Piripiri (PI), diferentes métodos de estimativa da evapotranspiração de referência (Hargreaves-Samani, Ivanov, Jensen-Haise, Thornthwaite e Priestley-Taylor), comparando-os com o método-padrão de Penman-Monteith-FAO. Foi utilizado um conjunto de dados diários de dez anos (2002 a 2012) de temperatura do ar, velocidade do vento, umidade relativa do ar e insolação. Os dados foram obtidos na estação meteorológica convencional do Instituto Nacional de Meteorologia (INMET), situada no município de Piripiri. A estimativa da ETo foi feita para o período chuvoso (janeiro a maio) e seco (junho a dezembro). Os resultados mostraram que o uso da equação de Jensen-Haise apresentou o melhor desempenho na estimativa diária da evapotranspiração de referência nas condições climáticas de Piripiri (PI) nas duas escalas de tempo avaliadas (chuvoso e seco).

Palavras-chave: Agricultura. Manejo da Irrigação. Agrometeorologia.

\section{Introdução}

As atividades agrícolas demandam grandes quantidades de água e, sendo cada vez mais preocupante a sua escassez, esforços têm sido empregados no desenvolvimento de pesquisas que possibilitem a sua economia em todo o planeta. Neste sentido, o correto conhecimento da evapotranspiração das culturas, seja para elaboração de projetos e/ou manejo de irrigação, assume fundamental importância.

A estimativa adequada da evapotranspiração da cultura (ETc) consiste no principal parâmetro a ser considerado no dimensionamento e manejo de sistemas de irrigação, uma vez que ela totaliza a quantidade de água utilizada nos processos de evaporação e transpiração pela cultura durante determinado período. De forma bastante simplificada, a ETc pode ser obtida por meio do valor da

1 Universidade Federal do Ceará, doutorando em Engenharia Agrícola (Irrigação e Drenagem). Fortaleza, Ceará, Brasil. alburibeiro@hotmail.com. Av. Mister Hull, s/n, Pici, Fortaleza (CE), CEP 60455-760.

2 Universidade Federal do Piauí - Campus Professora Cinobelina Elvas, mestre em Agronomia (Solos e Nutrição de Plantas). Bom Jesus, Piauí, Brasil. marcelosimeao16@gmail.com. Rodovia Municipal Bom Jesus - Viana, Km 01, Planalto Horizonte, Bom Jesus (PI), CEP 64900-000.

3 Universidade Federal Rural de Pernambuco, doutoranda em Fitopatologia. Recife, Pernambuco, Brasil. anandarbsantos@gmail.com. Rua Dom Manoel de Medeiros, s/n, Dois irmãos, Recife (PE), CEP: 52171-900. 
evapotranspiração de uma cultura de referência (ETo) corrigida pelo coeficiente da cultura (Kc), que depende do tipo de cultura e de seu estágio de desenvolvimento (SILVA; CAMPOS; AZEVEDO, 2009).

Existem vários métodos de estimativa da evapotranspiração de referência (ETo) disponíveis na literatura, a destacar: Jensen-Haise (PEREIRA; VILLA NOVA; SEDIYAMA, 1997), Ivanov (JENSEN, 1973), Thornthwaite (THORNTHWAITE; MATHER, 1948), Priestley-Taylor (PRIESTLEY; TAYLOR, 1972) e Penman-Monteith (ALLEN et al., 1998).

0 método de Penman-Monteith é considerado pela FAO como o padrão para estimativa da ETo (ALLEN et al., 1998). No entanto, para a sua utilização é necessário o conhecimento de variáveis meteorológicas, como temperatura do ar, umidade relativa do ar, radiação solar e velocidade do vento que nem sempre estão disponíveis para os produtores rurais, sendo necessária a utilização de métodos mais simples para o cálculo de ETo (ARAÚJO et al., 2011).

No município de Piripiri (PI), localiza-se o Perímetro Irrigado do Açude Caldeirão, com uma área irrigada de 398 ha, explorados principalmente com os cultivos de feijão-caupi e melancia. Para a região, o conhecimento de métodos simples e precisos de estimativa da ETo é fundamental para o manejo correto da irrigação pelos irrigantes do perímetro e demais produtores rurais.

No mesmo município, Silva (2012) verificou que a estimativa da ETo pelos métodos de Priestley-Taylor (PT) e de Thornthwaite, com temperatura efetiva (THtef), não diferiram estatisticamente do método-padrão de Penman-Monteith FAO. Em contrapartida, Nolêto et al. (2013) constataram que os métodos de Hargreaves-Samani e de Priestley-Taylor (PT) foram os que mais se aproximaram do método-padrão. Esses resultados contrastantes, notadamente por usarem séries de dados climáticos distintas, demonstram a importância da realização de novos estudos que recomendem melhor a estimativa da evapotranspiração de referência (ETo) no município, visando orientar o manejo da irrigação no Perímetro Irrigado do Açude Caldeirão.

Diante do exposto, objetivou-se com a realização deste trabalho avaliar o desempenho de métodos para a estimativa da ETo: Hargreaves-Samani, Ivanov, Jensen-Haise, Thornthwaite e Priestley-Taylor no período chuvoso e seco, comparando-os com o método-padrão de Penman-Monteith FAO em Piripiri, visando orientar melhor o manejo da irrigação no Perímetro Irrigado do Açude Caldeirão.

\section{Materiais e métodos}

O trabalho foi realizado utilizando dados meteorológicos obtidos entre o período de $1^{\circ}$ de janeiro de 2002 a 31 de dezembro de 2012, na estação meteorológica convencional do Instituto Nacional de Meteorologia (INMET), situada no município de Piripiri (04² 20' 00,4" S; 41 43' 52" W; Alt. 164 m). De acordo com a classificação climática de Köppen (1948), o clima da região é caracterizado como Aw', com precipitação média anual de 1.400 mm, máxima de 1.600 mm e mínima de 1.000 mm.

Foram utilizados dados diários de temperaturas máxima (Tmáx), média (Tméd) e mínima (Tmín) do ar, insolação, umidade relativa média do ar (URméd) e velocidade do vento a $10 \mathrm{~m}$ de altura, sendo posteriormente convertida para $2 \mathrm{~m}$. A partir dos dados de insolação, estimou-se a radiação solar global, utilizando a equação de Angström - Prescott (ALLEN et al., 1998).

Os dados analisados foram divididos em dois períodos: seco e chuvoso. Estabeleceu-se como período seco o período compreendido pelos meses de junho a dezembro e o período chuvoso entre os meses de janeiro a maio (Tabela 1 ). Os dias em que houve falhas nos dados foram excluídos. 
Tabela 1. Períodos selecionados com seus respectivos anos, meses e dias com disponibilidade de dados para a cidade de Piripiri (PI).

\begin{tabular}{ccccc}
\hline Estação & Período & Anos & N $^{\circ}$ de meses & Dias com dados disponíveis \\
\hline Piripiri & Chuvoso & 2002 a 2012 & 50 & 1.500 \\
& Seco & 2002 a 2012 & 70 & 2.100 \\
\hline
\end{tabular}

De posse dos dados, realizou-se a estimativa da evapotranspiração de referência pelos métodos de Penman-Monteith-FAO (padrão), Hargreaves-Samani, Ivanov, Camargo, Thornthwaite, Jensen-Haise e Priestley-Taylor.

A evapotranspiração de referência (ETo) pelo método de Penman-Monteith-FAO (considerado padrão) (ALLEN et al.,1998) foi obtida pela equação abaixo:

$$
E T o=\frac{0,408 \Delta(R n-G)+\gamma\left(\frac{900}{(\text { Tméd }+273)}\right) U_{2}\left(e_{s}-e_{a}\right)}{\Delta+\gamma\left(1+0,34 U_{2}\right)}
$$

Onde: ETo: evapotranspiração de referência ( $m m$ dia-1); Rn: saldo de radiação à superfície da cultura (MJ $m-2$ dia-1); G: fluxo de calor no solo (MJ m-2 dia-1); Tméd: temperatura do ar $\left({ }^{\circ} \mathrm{C}\right)$; U2: velocidade do vento à altura de 2 m (m s-1); es: pressão de saturação do ar (kPa); ea: pressão de vapor atual do ar (kPa); (es - ea): déficit de pressão de vapor (kPa); $\Delta$ : declividade da curva de pressão de vapor de saturação $\left(\mathrm{kPa}{ }^{\circ} \mathrm{C}-1\right)$ e $\mathrm{Y}$ : constante psicrométrica $\left(0,0677 \mathrm{kPa}{ }^{\circ} \mathrm{C}-1\right)$.

O método de Hargreaves-Samani (PEREIRA; VILLA NOVA; SEDIYAMA, 1997) foi utilizado, conforme a equação:

ETo $=0,0023 R a\left(\operatorname{Tmax}_{c}-\operatorname{Tmin}_{c}\right) 0,5($ Tméd $+17,8)$

Sendo: Ra: radiação solar extraterestre $\left(\mathrm{mm} \mathrm{dia}^{-1}\right) ; \operatorname{Tmax}_{\mathrm{c}}$ : temperatura máxima do $\operatorname{ar}\left({ }^{\circ} \mathrm{C}\right) ; \operatorname{Tmin}_{\mathrm{c}}$ : temperatura mínima do ar $\left({ }^{\circ} \mathrm{C}\right)$ e Tméd: temperatura média do $\operatorname{ar}\left({ }^{\circ} \mathrm{C}\right)$.

Realizou-se a estimativa da ETo pelo método de Ivanov (Jensen, 1973) por meio da equação (03):

ETo $=0,006\left(25+T_{m e ́ d}^{2}\left(1-\frac{\text { URméd }}{100}\right)\right.$

Em que Tméd é a temperatura média em ${ }^{\circ} \mathrm{C}$ e URméd é a umidade relativa do ar em \%.

O método de Camargo (1971) foi utilizado conforme a equação (04):

ETo $=0,01$ RaTmédK

Em que: $\mathrm{Ra}$ = radiação solar extraterrestre incidente acima da atmosfera no dia 15 de cada mês ( $\mathrm{mm} \mathrm{d}^{-1}$ ). Para a obtenção de Ra em $\mathrm{mm} \mathrm{d}^{-1}$, dividem-se os valores em $\mathrm{MJm}^{-2} \mathrm{~d}^{-1}$ por 2,45; Tméd é a temperatura média do ar em ${ }^{\circ} \mathrm{C}$ e $\mathrm{K}=$ fator de ajuste de Camargo.

A estimativa da ETo diária pelo método de Thornthwaite (1948) foi realizada por meio das equações 05, 06, 07, 08 e 09:

$\mathrm{ETo}=\frac{\mathrm{ETp}}{30} \times \frac{\mathrm{N}}{12}$

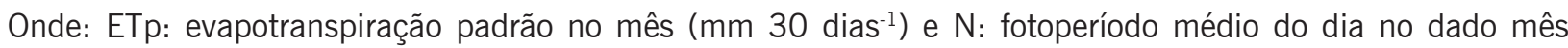
(condição $=12$ horas).

Para o cálculo de ETp, foram utilizadas as equações a seguir:

ETp $=16\left(\frac{10 \mathrm{Tméd}}{\mathrm{I}}\right)^{\mathrm{a}} \quad$ para $0^{\circ} \mathrm{C} \leq \mathrm{TMéd}<26,5^{\circ} \mathrm{C}$

ETp $=-415,85+32,24$ Tméd $-0,43 T^{2} \quad$ para Tméd $\geq 26,5^{\circ} \mathrm{C}$ 
$\mathrm{I}=12(0,2 \mathrm{Tméd})^{1,514} \quad$ para Tméd $>0^{\circ} \mathrm{C}$

$a=0,4924+1,79 \times 10^{2} \mathrm{I}-7,71 \times 10^{-5} \mathrm{I}^{2}+6,75 \times 10^{-7} \mathrm{I}^{3}$

Sendo: Tméd: média de temperatura para o dia ou período $\left({ }^{\circ} \mathrm{C}\right)$; I e a: índices térmicos regionais e Ta: normal climatológica da temperatura anual.

O método de Jensen-Haise (PEREIRA; VILLA NOVA; SEDIYAMA, 1997) foi utilizado conforme a equação (10):

ETo $=\operatorname{Rs}(0,025$ Tméd $+0,008)$

em que: Tméd = temperatura média diária, ${ }^{\circ} \mathrm{C}$; Rs = radiação solar global convertida em unidades de água evaporada, $\mathrm{mm}$.

Pelo método de Priestley-Taylor (1972) a ETo em mm.dia-1 foi obtida pela equação (13):

$\mathrm{ETo}=1,26 \mathrm{~W}\left(\frac{\mathrm{Rn}-\mathrm{G}}{\lambda}\right)$

Sendo: $\lambda$ : calor latente de vaporização a $20^{\circ} \mathrm{C}\left(2,45 \mathrm{MJ} \mathrm{kg}^{-1}\right)$; W: fator de ponderação em função da temperatura (T) e do coeficiente psicrométrico. Pode ser calculado segundo as seguintes equações:

$\mathrm{W}=0,407+0,0145 \mathrm{~T} \quad\left(0^{\circ} \mathrm{C}<\mathrm{T}<16^{\circ} \mathrm{C}\right)$

$\mathrm{W}=0,483+0,01 \mathrm{~T} \quad\left(16,1^{\circ} \mathrm{C}<\mathrm{T}<32^{\circ} \mathrm{C}\right)$

Para a análise comparativa entre os métodos avaliados e método-padrão de PM, foram realizados cálculos de erro-padrão de estimativa (EEP) (equação 14), dos coeficientes de determinação $\left(R^{2}\right)$ e correlação $(r)$ (equação 15), o índice d (Equação 16) proposto por Willmott, Ckleson e Davis (1985) e o coeficiente $c$ de desempenho, que é o produto do coeficiente $r$, com o índice $d$ (CAMARGO; SENTELHAS, 1997).

EEP $=\frac{\sqrt{\sum_{i=1}^{n}(P i-O i)^{2}}}{n-1}$

em que: EEP = estimativa do erro-padrão, em mm. dia-1; $\mathrm{Oi}=$ valores estimados pelo método-padrão, PenmanMonteith, em mm.dia-1 $\mathrm{Pi}=$ corresponde aos valores estimados pelos métodos, em $\mathrm{mm}$. dia-1 $; \mathrm{n}=$ número de observações.

$d=1-\left[\frac{\sum(P i-O i)^{2}}{\left.\sum(\mid \mathrm{Pi}-\bar{O})|+| O i-\bar{O} \mid\right)^{2}}\right]$

em que: $r$ = índice de precisão ou coeficiente de correlação; $d$ : índice de Willmott; Pi: valores de ETo estimados pelos métodos não padrão ( $\left.\mathrm{mm} \mathrm{dia}^{-1}\right)$; Oi: valores de ETo estimados pelo método-padrão $(\mathrm{mm}$.dia-1) e $\overline{\mathrm{O}}$ : valores médios total do período em que ETo foi estimado pelo método-padrão (mm.dia-1).

De acordo com Camargo e Sentelhas (1997), calculou-se o coeficiente de desempenho ou de confiança de cada método para estimar ETo em relação ao método-padrão, descrito na equação abaixo:

c: $r \times d$

Onde: c: coeficiente de desempenho ou de confiança (Tabela 2); r: correlação e d: índice de Willmott. 
Tabela 2. Classificação do desempenho conforme o índice de confiança "c"

\begin{tabular}{ll}
\hline Valor de c & Desempenho \\
\hline$\geq 0,85$ & Ótimo \\
0,76 a 0,85 & Muito bom \\
0,66 a 0,76 & Bom \\
0,61 a 0,65 & Mediano \\
0,51 a 0,60 & Sofrível \\
0,41 a 0,50 & Mau \\
$\leq 0,40$ & Péssimo \\
\hline
\end{tabular}

Fonte: Camargo; Sentelhas (1997).

\section{Resultados e discussões}

\section{Período chuvoso}

No período chuvoso, os melhores métodos entre os estudados foram os de Jensen-Haise $(\mathrm{JH})$ com desempenho Ótimo ( $c=0,90 ; d=0,98$; EEP $=0,91 \mathrm{~mm} \cdot \mathrm{dia}^{-1}$ ) e de Priestley-Taylor (PT) com desempenho Muito Bom ( $c=0,77 ; d=0,98$; EEP $=0,94$ mm.dia ${ }^{-1}$ ), porém com estimativa de erro-padrão (EEP) maior em relação a Jensen-Haise (JH), implicando na aplicação de maiores lâminas de irrigação (Tabela 3).

Tabela 3. Coeficiente de determinação $\left(R^{2}\right)$, coeficiente de correlação ( $r$ ), estimativa do erro-padrão (EEP), índice de concordância (d) e índice de confiança ou desempenho (c) nas escalas diárias para o período chuvoso (janeiro a maio) para Piripiri, Piauí.

\begin{tabular}{ccccccc}
\hline Método & EEP & $\mathbf{R}^{2}$ & $\mathbf{r}$ & $\mathbf{d}$ & $\mathbf{c}$ & Desempenho \\
\hline HS & 0,90 & 0,39 & 0,62 & 0,88 & 0,54 & Sofrível \\
IVN & 1,25 & 0,40 & 0,63 & 0,90 & 0,56 & Sofrível \\
JH & 0,91 & 0,85 & 0,92 & 0,98 & 0,90 & Ótimo \\
TW & 0,98 & 0,18 & 0,42 & 0,90 & 0,38 & Péssimo \\
PT & 0,94 & 0,63 & 0,79 & 0,98 & 0,77 & Muito Bom \\
\hline
\end{tabular}

$\mathrm{HS}=$ método de Hargreaves-Samani; IVN = método de Ivanov; JH = método de Jensen-Haise; PT = método de Priestley-Taylor; EEP = estimativa do erro padrão; $R^{2}=$ coeficiente de determinação; $r=$ coeficiente de correlação; $d$ = coeficiente de concordância; $c$ = coeficiente de desempenho.

O melhor desempenho do método de Jensen-Haise justifica-se em virtude de o mesmo ter sido desenvolvido para a aplicação nas áreas irrigadas de regiões áridas e semiáridas, semelhante às condições climáticas encontradas na área onde este estudo foi desenvolvido (MEDEIROS, 2008). 0 desempenho "Muito Bom" do método de Priestley-Taylor (PT) justifica-se em virtude da baixa advecção, por tratar-se do período chuvoso.

Em estudo conduzido por Gonçalves et al. (2009), para o município de Sobral, CE, o método de Jensen-Haise apresentou índice de desempenho "c" classificado como "Bom", apresentando índice de desempenho de 0,69, coeficiente de correlação "r" de 0,76 e estimativa de erro-padrão (EEP) de 0,97 mm dia-1. Em Uberlândia (MG), Silva et al. (2011), usando o método de Priestley-Taylor, obtiveram índice de desempenho classificado como "Ótimo".

Os métodos de Hargreaves-Samani (HS) e Ivanov (IVN) apresentaram desempenho caracterizado como "Sofrível", enquanto que o de Thornthwaite (TW), com índice c = 0,38, foi caracterizado como "Péssimo". O método de Hargreaves-Samani foi obtido para a condição semiárida. Assim, seu 
desempenho apresenta uma redução na precisão da estimativa da evapotranspiração nos meses chuvosos (ALENCAR et al., 2011).

Resultados semelhantes para o método de Hargreaves-Samani (HS) foram observados por Araújo, Costa e Santos (2007), quando analisaram as estimativas de ETo pelos métodos de Thornthwaite, Hargreaves-Samani, Blaney-Criddle, Penman-Monteith-FAO, Tanque Classe "A" e Makkink, para o município de Boa Vista (RR), que encontraram $c=0,57$, classificado como "Sofrível". Gonçalves et al. (2009) obtiveram para Sobral (CE), pelo método de Ivanov, valor do índice de desempenho "c" classificado também como "Sofrível".

O péssimo desempenho do método de Thornthwaite $(\mathrm{TH})$ justifica-se em virtude de ele ter sido desenvolvido para estimativa de ETo em escala mensal, enquanto que no presente estudo, as estimativas de ETo foram efetuadas na escala diária. Silva et al. (2005), em Campina Grande (PB), também observaram que o método de Thornthwaite (TW) não apresentou resultados satisfatórios na estimativa da ETo.

A Figura 1 apresenta as regressões lineares entre valores diários de evapotranspiração de referência estimados pelos métodos estudados em função do método de referência de Penman-Monteith-FAO 56 para o período chuvoso. Observa-se que o método de Ivanov (IVN) subestimou os valores de ETo estimado pelo método-padrão, os de Priestley-Taylor (PT) e Jensen-Haise (JH) superestimaram a ETo, enquanto que os de Hargreaves-Samani e Thorthwaite apresentaram, ao longo do ano, estimativas que subestimaram e superestimaram os valores de ETo estimados pelo método-padrão.
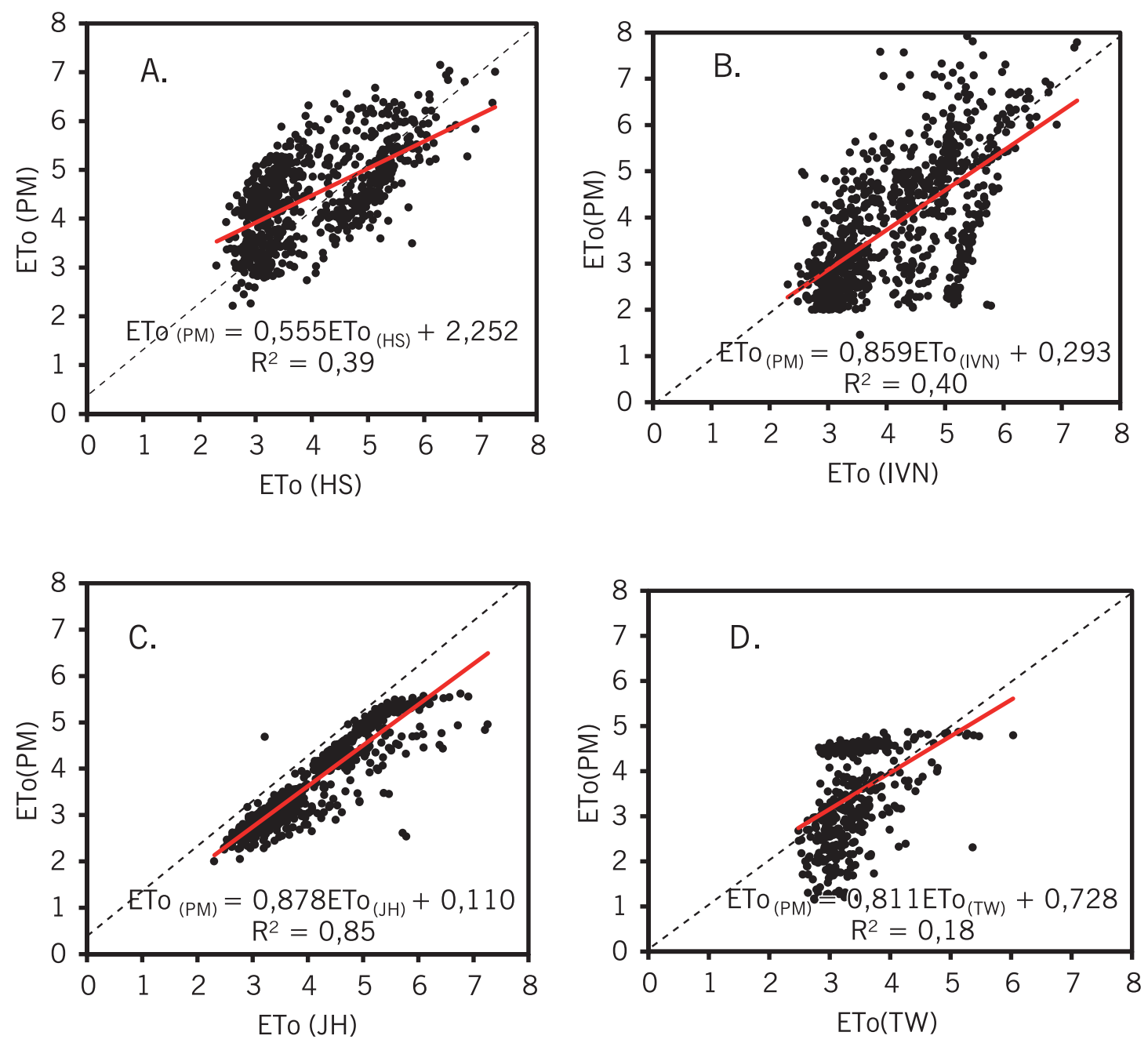


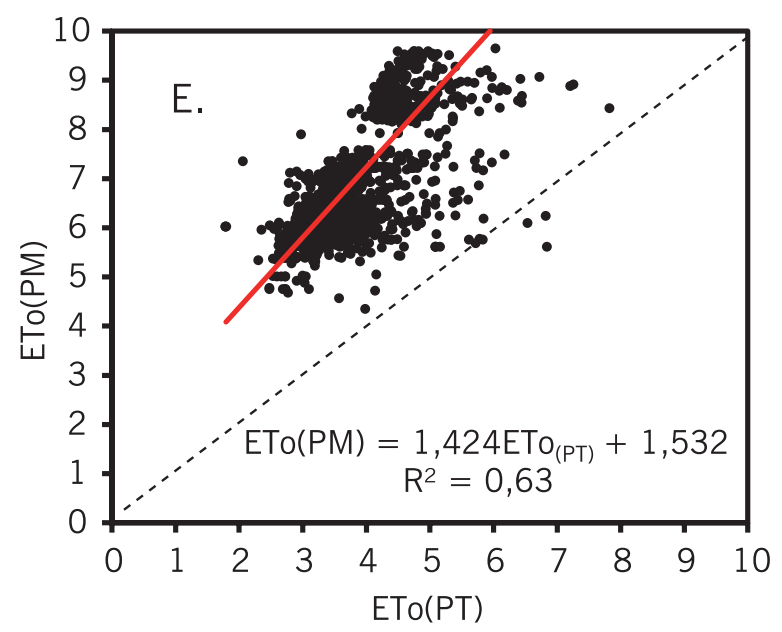

Figura 1. Regressão linear entre os valores diários de evapotranspiração de referência (ETo) estimados pela comparação dos métodos de Hargreaves-Samani (A), Ivanov (B), Jensen-Haise (C), Thornthwaite (D) e PriestleyTaylor (E), com o método-padrão Penman-Monteith durante o período chuvoso, em Piripiri (PI).

O método de Priestley-Taylor, apesar de ter obtido desempenho "Muito Bom", apresentou valores de ETo que se distanciaram muito dos estimados pelo método-padrão, não sendo assim recomendado para o manejo da irrigação no município de Piripiri (PI) durante o período chuvoso. Silva (2012), em um estudo de estimativa da ETo em 19 cidades do estado do Piauí, verificou que em Piripiri, durante o período chuvoso, o método de Priestley-Taylor (PT) também superestimou a ETo em relação ao método de PM-FAO.

O método de Jensen-Haise $(\mathrm{JH})$ foi o que apresentou valores mais próximos aos estimados pelo método-padrão, sendo o método mais recomendado para o manejo da irrigação no município durante o período chuvoso.

\section{Período seco}

No período seco, o método com melhor desempenho entre os estudados foi o de Jensen-Haise $(\mathrm{JH})$, com desempenho Ótimo ( $c=0,89 ; \mathrm{d}=0,98$; EEP $=1,08 \mathrm{~mm} \cdot \mathrm{dia}^{-1}$ ), continuando, portanto, com o mesmo desempenho verificado para o período chuvoso. Na sequência, aparecem os métodos de Hargreaves-Samani (HS), Ivanov (IVN) e Priesley-Taylor (PT), todos com desempenho considerado Mediano (Tabela 4).

Tabela 4. Coeficiente de determinação $\left(R^{2}\right)$, coeficiente de correlação $(r)$, estimativa do erro padrão (EEP), índice de concordância (d) e índice de confiança ou desempenho (c) na escala diária para o período seco (junho a dezembro) para Piripiri, Piauí

\begin{tabular}{ccccccc}
\hline Métodos & EEP & $\mathbf{R}^{\mathbf{2}}$ & $\mathbf{r}$ & $\mathbf{d}$ & $\mathbf{c}$ & Desempenho \\
\hline HS & 1,01 & 0,43 & 0,65 & 0,94 & 0,61 & Mediano \\
IVN & 1,38 & 0,46 & 0,67 & 0,97 & 0,65 & Mediano \\
JH & 1,08 & 0,84 & 0,91 & 0,98 & 0,89 & Ótimo \\
TW & 1,00 & 0,11 & 0,34 & 0,91 & 0,31 & Péssimo \\
PT & 1,83 & 0,41 & 0,64 & 0,99 & 0,63 & Mediano \\
\hline
\end{tabular}

$\mathrm{HS}=$ método de Hargreaves-Samani; IVN = método de Ivanov; JH = método de Jensen-Haise; TW = Thorntwaite; $\mathrm{PT}=$ método de Priestley-Taylor; EEP = estimativa do erro padrão; $\mathrm{R}^{2}=$ coeficiente de determinação; $r=$ coeficiente de correlação; $d$ = coeficiente de concordância; $c$ = coeficiente de desempenho. 
Os métodos de Hargreaves-Samani (HS) e Ivanov (IVN) melhoraram seus desempenhos em relação ao período chuvoso. Esse comportamento justifica-se em virtude das elevadas temperaturas no período. 0 método de Priestley-Taylor apresentou desempenho inferior se comparado ao período chuvoso, devido ao aumento da advecção no período seco.

Silva (2012) observou que, em Piripiri (PI), no período seco, os métodos de Hargreaves-Samani e Priestley-Taylor apresentaram desempenho superior quando comparado ao período chuvoso, o que também foi constatado por Alencar et al. (2011) para Uberaba(MG), em relação ao método de Hargreaves-Samani (HS).

Com relação ao método de Thornthwaite (TW), verificou-se que esse continuou apresentando o pior desempenho. Resultados semelhantes foram encontrados por Borges e Mendiondo (2007) para a bacia do Rio Jacupiranga (SP).

A Figura 2 apresenta as regressões lineares entre valores diários de evapotranspiração de referência estimados pelos métodos estudados em função do método de referência de Penman-Monteith-FAO 56 para o período seco. Observa-se que os métodos de Ivanov (IVN) e Priestley-Taylor (PT) superestimaram os valores de ETo estimado pelo método-padrão, o de Jensen-Haise $(\mathrm{JH})$ subestimou a ETo, enquanto que os de Thornthwaite (TW) e Hargreaves-Samani apresentaram, ao longo do ano, estimativas que subestimaram e superestimaram os valores de ETo estimados pelo método-padrão.
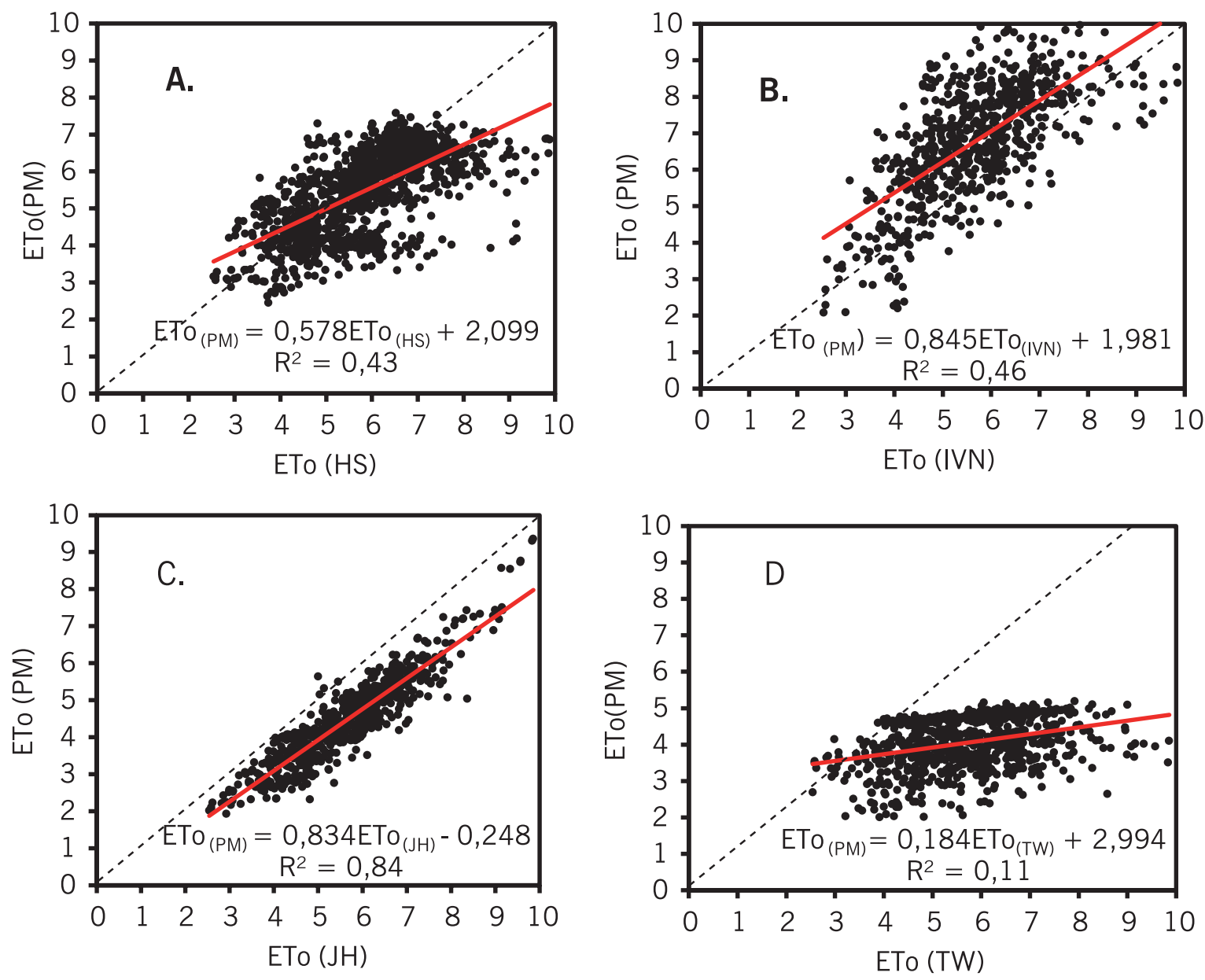


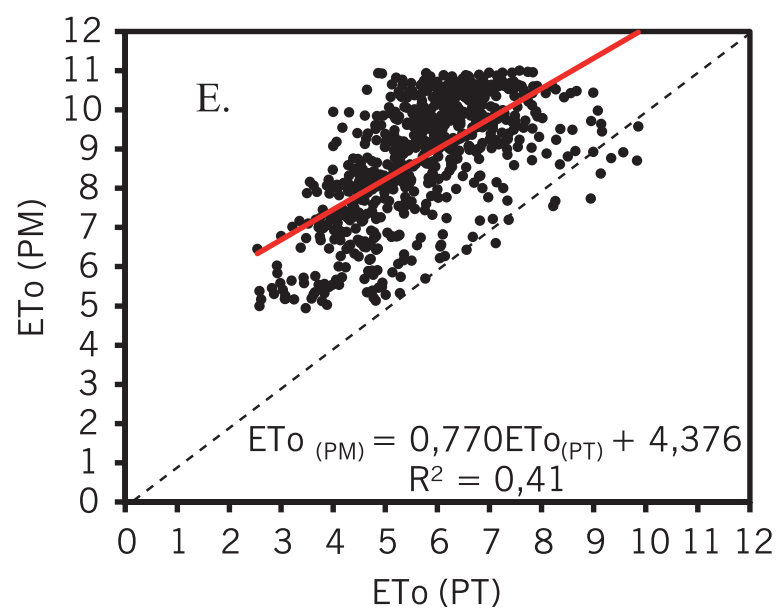

Figura 2. Regressão linear entre os valores diários de evapotranspiração de referência (ETo) estimados pela comparação dos métodos de Hargreaves-Samani (A), Ivanovit (B), Jensen-Haise (C), Thornthwaite (D) e Priestley-Taylor (E), com o método-padrão, Penman-Monteith, durante o período seco, em Piripiri (PI).

Os valores de ETo estimados pelo método de Priestley-Taylor novamente se distanciaram muito dos obtidos pelo método-padrão. O método de Priestley-Taylor também não estimou satisfatoriamente a ETo em estudo realizado por Barros et al. (2009) para a região de Seropédica (RJ) e Araújo et al. (2010) para as cidades de Crateús e Jaguaruana, ambas no Ceará, contrariando os resultados obtidos por Fanaya Júnior et al., (2012), Guedes Filho et al. (2011) e Reis, Bragança e Garcia (2007), em Aquidauana (MS), Areia (PB) e regiões norte, sul e serrana do estado do Espírito Santo, respectivamente.

Para o período chuvoso, o método de Jensen-Haise, além de ter apresentado o melhor desempenho, obteve valores de ETo bem próximos dos estimados pelo método-padrão, sendo o método mais recomendado para o manejo da irrigação no município de Piripiri (PI) também no período seco.

Resultados semelhantes foram obtidos por Pereira et al. (2009) na região da Serra da Mantiqueira, em Minas Gerais, ao constatarem que o método de Jensen-Haise (JH) foi um dos mais adequados para estimar a ETo, independentemente da época do ano, além de ter apresentado o melhor ajuste.

O uso de métodos de estimativa da evapotranspiração que utilizam poucas variáveis, como é o caso do método de Jensen-Haise, que tem como parâmetros de entrada apenas a temperatura média diária e a radiação solar, que podem ser facilmente obtidos pelos produtores, é de grande importância para o município de Piripiri, pois facilita o manejo da irrigação pelos produtores no Perímetro Irrigado Açude Caldeirão.

\section{Conclusões}

O uso da equação de Jensen-Haise apresentou o melhor desempenho na estimativa diária da evapotranspiração de referência nas condições climáticas de Piripiri (PI), em comparação ao método-padrão da FAO, nas duas escalas de tempo estudadas (chuvoso e seco). 


\section{Comparison of methods for estimating reference evapotranspiration in dry and wet seasons to Piripiri (PI)}

\section{Abstract}

Several empirical methods have been used to quantify the reference evapotranspiration because of difficulties for producers to access the variables used by the Penman-Monteith-FAO standard. Therefore, this study aimed to evaluate, for the climate conditions of Piripiri (PI), different methods for estimating the reference evapotranspiration (Hargreaves-Samani, Ivanov, Jensen-Haise, Thornthwaite and Priestley-Taylor), comparing them to the Penman-Monteith-FAO standard. A set of ten years daily data for air temperature, wind speed, relative humidity and heat stroke was used (2002-2012). Data were obtained from the conventional weather station of the National Institute of Meteorology (INMET), located in Piripiri. The ETo estimate was carried out for the wet season (January-May) and the dry season (June-December). The results showed that the use of Jensen-Haise equation presented the best performance for daily estimation of reference evapotranspiration in the climate conditions of Piripiri, in both timescales evaluated (wet and dry).

Keywords: Agriculture. Irrigation management. Agrometeorology.

\section{Referências}

ALENCAR, L. P.; DELGADO, R. C.; ALMEIDA, T. H.; WANDERLEY, H. S. Comparação de diferentes métodos de estimativa diária da evapotranspiração de referência para a região de Uberaba. Revista Brasileira de Ciências Agrárias, Recife, v. 6, n. 2, p. 337-343, 2011.

ALLEN, R. G.; PEREIRA, L. S.; RAES, D.; SMITH, M. Crop evapotranspiration: guidelines for computing crop water requirements. Roma: FAO, 1998. 300 p.

ARAÚJO, W. F.; COSTA, A. A.; SANTOS, A. E. dos. Comparação entre métodos de estimativas da evapotranspiração de referência (ETo) para Boa Vista- RR. Revista Caatinga, v. 20, n. 4, p. 84 - 88 , 2007.

ARAÚJO, E. M.; OliVEIRA, J. B.; ARAúJO, E. M.; LEDO, E. R. F.; SILVA, M. G. Desempenho de métodos de estimativa de ETo correlacionados com a equação padrão Penman-Monteith FAO-56, em cidades do estado do Ceará. Revista ACTA Tecnológica, Iguatu, v. 5, n. 2, 18p. 2010.

ARAÚJO, G. L.; REIS, E. F.; MARTINS, C. A. S.; BARBOSA, V. S.; RODRIGUES, R. R. Desempenho comparativo de métodos para a estimativa da evapotranspiração de referência (ETo). Revista Brasileira de Agricultura Irrigada, Fortaleza, v. 5, n. 2, p.84-95, 2011.

BARROS, V. R.; SOUZA, A. P.; FONSECA, D. C.; SILVA, L. B. D. Avaliação da evapotranspiração de referência na região de Seropédica, Rio de Janeiro, utilizando lisímetro de pesagem e modelos matemáticos. Revista Brasileira de Ciências Agrárias, Recife, v. 4, n. 2, p. 198-203, abr./jun. 2009.

BORGES, A. C. de; MENDIONDO, E. M. Comparação entre equações empíricas para estimativa da evapotranspiração de referência na Bacia do Rio Jacupiranga. Revista Brasileira de Engenharia Agrícola e Ambiental, Campina Grande, v. 11, n. 3, p. 293-300, 2007. 
CAMARGO, A. P.; SENTELHAS, P. C. Avaliação do desempenho de diferentes métodos de estimativa da evapotranspiração potencial no Estado de São Paulo. Revista Brasileira de Agrometeorologia, Santa Maria, v. 5, n. 1, p. 89-97, 1997.

FANAYA JÚNIOR, E. D.; LOPES, A. S.; OLIVEIRA, G. Q.; JUNG, L. H. Métodos empíricos para estimativa da evapotranspiração de referência para Aquidauana, MS. Irriga, Botucatu, v. 17, n. 4, p. 418-434, 2012.

GUEDES FILHO, D. H.; SANTOS JUNIOR, J. A.; COSTA FILHO, J. F.; FRANCISCO, P. R. M.; CAMPOS, V. B. Estimativa da evapotranspiração de referência para a cidade de Areia, Paraíba. Revista Brasileira de Agricultura Irrigada, Fortaleza, v.5, n.1 p.37-47, 2011.

GONCALVES, F. M.; FEITOSA, H. O.; CARVALHO, C. M.; GOMES FILHO, R. R.; JÚNIOR, M. V. Comparação de métodos da estimativa da evapotranspiração de referência para o município de SobralCE. Revista Brasileira de Agricultura Irrigada, Fortaleza, v. 3, n. 2, p. 71-77, 2009.

JENSEN, ME (ed.). Consumptive use of water and irrigation water requirements. American Society of Civil Engineers, New York, USA, 215p. 1973.

MEDEIROS, P. V. Análise da evapotranspiração de referência a partir de medidas lisimétricas e ajuste estatístico de estimativas de nove equações empiríco-teóricas com base na equação de Penman-Monteith. 2008. 241p. Dissertação (Mestrado em Hidráulica e Saneamento), Universidade de São Paulo, São Carlos, 2008.

NOLÊTO, D. H.; ANDRADE JÚNIOR, A. S; BRAGA, D. L.; MOUSINHO, F. E. P.; SOUSA, C. C Comparação de métodos empíricos de estimativa da evapotranspiração de referência para Piripiri, PI. In: CONGRESSO BRASILEIRO DE ENGENHARIA AGRÍCOLA, 12., 2013, Fortaleza. Anais... Fortaleza, 2013.

REIS, E. F. de; BRAGANÇA, R. de; GARCIA, G. O. Estudo comparativo da estimativa da evapotranspiração de referência para três localidades do Estado do Espírito Santo no período seco. Idesia, Arica, v. 25, n. 3, p. 75-7854, 2007.

SILVA, C. O. Avaliação de métodos para estimativa da evapotranspiração de referência no estado do Piauí. 2012. 121f. Dissertação (Mestrado em Engenharia Agrícola), Universidade Federal do Ceará, Fortaleza, 2012.

SILVA, V. P. R.; CAMPOS, J. H. B. C.; AZEVEDO, P. V. de. Wateruse efficiency and evapotranspiration of mango orchard grown in northeastern region of Brazil. Scientia Horticulturae, v. 1, n. 120, p. 467-472, 2009.

SILVA, V. J.; CARVALHO, H. P.; SILVA, C. R.; CAMARGO, R.; TEODORO, R. E. F. Desempenho de diferentes métodos de estimativa da evapotranspiração de referência diária em Uberlândia, MG. Bioscience Journal, Uberlândia, v. 27, n. 1, p. 95-101, 2011.

SILVA, V. P. R.; BELO FILHO, A. F.; SILVA, B. B.; CAMPOS, J. H. B. C. Desenvolvimento de um sistema de estimativa da evapotranspiração de referência. Revista Brasileira de Engenharia Agrícola e Ambiental, Campina Grande, v. 9, n. 4, p. 547-553, 2005. 
PEREIRA, A. R.; VILLA NOVA, N. A.; SEDIYAMA, G. C. Evapotranspiração. Piracicaba: FEALQ, 1997. 183p.

PEREIRA, D. R.; YANAGE, S. N. M.; MELLO, C. R.; SILVA, A. M.; SILVA, L. A. Desempenho de métodos de estimativa da evapotranspiração de referência para a região da Serra da Mantiqueira, MG.

Ciência Rural, Santa Maria, v. 39, n. 9, p. 2488-2493, dez./2009.

PRIESTLEY, C. H. B.; TAYLOR, R. J. On the assessment of surface heat flux and evaporation using large-scale parameters. Monthly Weather Review, Madson, v. 100, p. 81- 92, 1972.

THORNTHWAITE, C. W.; MATHER, J. R. An approach toward a rational classification of climate. The Geographycal Review, New York, v. 38 n. 1, p. 55-94, 1948.

WILLMOTT, C. J., CKLESON, S. G., DAVIS, R. E. Statistics for the evaluation and comparison of models. Journal of Geophysical Research. Ottawa, v. 90, n. C5, p. 8995 - 9005, 1985.

\section{Histórico editorial}

Submetido em: 18/06/2015

Aceito em: 15/10/2015 